\title{
Evolution of Mitochondrial-Derived Peptides, Humanin and MOTSc and Changes in Insulin Sensitivity During Early Gestation
}

\section{David Ruiz}

Marqués de Valdecilla University Hospital

\section{Miguel Santibáñez}

University of Cantabria

\section{Bernardo Alio-Lavín}

Marqués de Valdecilla University Hospital

\section{Isabel Álvarez}

Marqués de Valdecilla University Hospital

\section{Ana Berja}

Marqués de Valdecilla University Hospital

\section{Coral Montalbán}

Marqués de Valdecilla University Hospital

Luis Alberto Vázquez ( $\sim$ luisvazquezsalvi@gmail.com )

University of Cantabria

\section{Research Article}

Keywords: mitochondrial-derived peptides, humanin, MOTSc, gestational diabetes mellitus, HOMA-IR, insulin resistance

Posted Date: June 10th, 2021

DOl: https://doi.org/10.21203/rs.3.rs-588198/v1

License: (a) (1) This work is licensed under a Creative Commons Attribution 4.0 International License. Read Full License 


\section{Abstract}

Aims: To study the evolution of mitochondrial-derived peptides (MDPs) and their relationship with changes in insulin sensitivity from the early stages of pregnancy.

Methods: MDPs (humanin and MOTSc) were assessed in the first and second trimesters of gestation in 28 pregnant women with gestational diabetes mellitus (GDM) and a subgroup of 45 matched pregnant women without GDM. Insulin resistance (IR) was defined as a HOMA-IR index $\geq 70$ percentile.

Results: We observed a significant reduction in both humanin and MOTSc levels from the first to the second trimesters of pregnancy. After adjusting for BMI we did not find an association between low levels of humanin and the occurrence of a high HOMA-IR index (adjusted OR= 2.63 and 3.14, linear p-trend 0.260 and 0.175 , respectively). Regarding MOTSc, an association was found only for the second trimester: adjusted $\mathrm{OR}=7.68$ (95\% $\mathrm{Cl} 1.49-39.67)$, linear $p$-trend=0.012. No significant associations were observed in humanin change with insulin resistance throughout pregnancy, but changes in MOTSc levels were significantly associated with HOMA-IR index: adjusted OR 3.73 (95\% Cl 1.03-13.50).

Conclusions: MOTSc levels, especially a strong decrease from the first to the second trimester of gestation, may be involved in increasing insulin resistance during early gestation.

\section{CLINICAL TRIAL REGISTRATION}

Not applicable

\section{Introduction}

Gestational diabetes mellitus (GDM) is one of the most important complications associated with pregnancy. Its onset is related to defects in insulin secretion, insulin sensitivity or a combination of both, and these conditions may become apparent early in pregnancy [1]. However, international guidelines recommend screening for GDM only late in the second trimester [2-4]. Despite this recommendation, nearly one-third of GDM diagnoses can occur before the 24th week, and these pregnant women with "early GDM" are at increased risk of complications than pregnant women with "late GDM" [5]. It is therefore essential to identify mediators that might play a role in the changes in insulin sensitivity from the early stages of gestation and to determine their predictive capacity for the occurrence of GDM.

Mitochondria are essential organelles for multiple aspects of cellular homeostasis. Mitochondrial dysfunction is implicated as a major contributing factor for a number of noncommunicable chronic diseases, including insulin resistance [6]. Moreover, impaired mitochondrial function may play a role in the risk of developing GDM; however, it is not known whether this dysfunction results in a primary defect in the pathophysiology of the disease [7]. Mitochondria have their own circular genome (mitochondrial DNA, mtDNA) of approximately 16.5 Kilobases comprise 37 genes that encode 13 proteins of the respiratory chain. However, in addition to their well-known function in cellular bioenergetics, different 
mitochondrial-derived peptides (MDPs), which are small bioactive peptides encoded by short open reading frames (sORFs) in mtDNA, have been identified in the last few years [8]. To date, 8 different MDPs have been described, acting as signaling agents in cytoprotection and energy regulation tasks. Humanin, a 24 aa polypeptide encoded by the 16S rRNA coding region of mtDNA, has been associated with functions related to increased cell survival and decreased apoptosis or oxidative stress. On the other hand, the mitochondrial open reading frame of 12S rRNA type-c (MOTSc) encoded by the 12S rDNA region of mtDNA is a 16 aa polypeptide whose functions are especially related to the enhancement of insulin sensitivity and the increase in beta-oxidation [9].

To our knowledge, no study has focused on the evolution of MDPs and their influences on changes in insulin sensitivity from the earliest stages of pregnancy.

\section{Subjects, Materials And Methods \\ 2.1. Study population}

The population of the present study was drawn from the one included in a previous study designed to establish the reference thresholds of thyroid function parameters in the pregnant population of our geographical area. The population was made up of healthy pregnant women who attended their first pregnancy control in the primary care clinics of area IV of Cantabria (Northern Spain). The criteria for inclusion were age $\geq 18$ years, first visit within the first trimester of pregnancy, absence of thyroid functional disorders and absence of chronic diseases (including diabetes). Exclusion criteria were having received fertility treatment and multiple gestations. Participants were invited to provide blood and urine samples in each of the gestation trimesters and to fill out a survey on sociodemographic aspects and dietary habits.

The initial sample included a total of 664 pregnant women. Forty-eight women were excluded because they experienced a miscarriage, 93 because they were found to have alterations in the parameters of thyroid function, and 55 because one of the samples from the first two trimesters were not available. Therefore, a total of 468 pregnant women without prepregnancy diabetes conformed to our final study sample. We identified all the pregnant women who were diagnosed with GDM that resulted in a total of $\mathrm{n}$ $=40(8.5 \%)$, and from the same study population, we selected a subgroup of matched controls by BMI, age, previous gestations and time of sampling (difference not exceeding three months). Finally, 12 pregnant women with GDM were excluded because we failed to identify matched controls, so the final study sample consisted of 28 pregnant women with GDM and 45 without diabetes. The number of controls was calculated to maintain a ratio greater than 1.5 controls for each GDM case.

\subsection{Data collection and biomarkers}

Through a structured questionnaire and the review of medical records, information was collected about maternal age, weight in the first and second trimesters, height, obstetric history and smoking habits. The $\mathrm{BMI}$ in the first and second trimesters was calculated as the weight in kilograms between the height in 
meters squared. GDM was diagnosed according to the usual protocol of our health service. This protocol consists of a universal screening in two steps: a nonfasting oral overload of $50 \mathrm{~g}$ in all pregnant women and if the blood glucose value at the hour is $\geq 140 \mathrm{mg} / \mathrm{dl}$, a diagnostic test was performed consisting of an oral glucose overload of $100 \mathrm{~g}$ with determination of fasting blood glucose and 1,2 and 3 hours later. A positive result was defined as 2 or more values above the thresholds established according to ADA criteria (NDDG): fasting $\geq 5.8 \mathrm{mmol} / \mathrm{l} ; 1-\mathrm{h}, \geq 10.6 \mathrm{mmol} / \mathrm{l} ; 2-\mathrm{h}, \geq 9.2 \mathrm{mmol} / \mathrm{l}$; and $3-\mathrm{h}, \geq 8.0 \mathrm{mmol} / \mathrm{I}^{5}$.

Blood samples were taken at 8:00 am while fasting between weeks 10-12 in the first trimester and between weeks $24-26$ in the second and then frozen at $-80^{\circ} \mathrm{C}$ until analysis. Humanin plasma was evaluated by ELISA (Humanin MT-RNR2) (Cusabio Biotech Co, LTD). Analytical sensitivity 7 pg/ml. Specificity: No cross reactions with humanin MT-RNR2 analogs were observed. The intra-assay reproducibility of the method is $<8 \%$, and the interassay reproducibility is $<10 \%$. The quantification of MOTSs was performed by ELISA (Cloud-Clone Corp, TX, USA). Analytical sensitivity $0.97 \mathrm{ng} / \mathrm{ml}$. Specificity: No cross reaction with MOTS-c analogs was observed. The intra-assay reproducibility of the method was $<10 \%$, and the interassay reproducibility was $<12 \%$. Glucose was determined automatically by the glucose oxidase method in an AtellicaTM CH Analyzer (Siemens Healthcare Diagnostics, Inc., Newark, DE, USA). The analytical sensitivity was $6 \mathrm{mg} / \mathrm{dl}$. The intra-assay reproducibility of the method was $<1.6 \%$, and the interassay reproducibility was $<4.2 \%$. Insulin was determined by automated immunoassay in an AtellicaTM IM Analyzer (Siemens Healthcare Diagnostics, Inc, Newark, DE, USA) Analytical sensitivity: $0.3 \mathrm{mIUI} / \mathrm{l}$; Intra-assay reproducibility of the method is $<1.8 \%$ and interassay $<3.6 \%$. All assays were performed without knowledge of case-control status.

We used the calculation of the homeostasis model assessment (HOMA) to evaluate both insulin resistance (HOMA-IR), according to the formula fasting serum insulin $(\mu \mathrm{U} / \mathrm{ml}) \times$ fasting plasma glucose $(\mathrm{mmol} / \mathrm{l}) / 22.5$, and beta cell function (HOMA- $\beta$ ), according to the formula $20 \mathrm{x}$ fasting insulin $(\mu \mathrm{U} / \mathrm{ml}) /$ fasting plasma glucose $(\mathrm{mmol} / \mathrm{l})-3.5$ in each trimester. We consider a high HOMA-IR those values $\geq 70$ th percentile.

\subsection{Statistical analysis}

Categorical variables are displayed as percentages, and the $\chi^{2 \text { test }}$ was used for comparisons between groups. Continuous variables are summarized as the mean \pm standard deviation (SD) or median (interquartile range), and comparisons between GDM cases and controls were performed by using Student's t-test in cases of normal distribution or the Mann-Whitney U-test when appropriate. For intragroup first- and second-trimester comparisons, we used the Related-Samples T Test or Wilcoxon Signed Rank test when appropriate. The correlation between humanin and MOTSc levels with age, BMI in each trimester and HOMA-IR as continuous variables was estimated through Spearman's correlation coefficient. To determine and compare the predictive capacity of each variable on the risk of a high HOMA-IR index or developing GDM, receiver operating characteristic (ROC) curves and the area under the curve (AUC) were determined. 
We used a multivariate logistic regression analysis to calculate the strength of the associations. MDPs were dichotomously categorized (high versus low values) according to the median and odds ratios (ORs) with $95 \%$ confidence intervals ( $95 \% \mathrm{Cls}$ ) adjusted for smoking status, body mass index (BMI) and age for a HOMA-IR $\geq$ p70 and adjusted for smoking status for GDM. In addition, when a significant association was identified, exposure-response trends (biological gradient, dose response pattern) were estimated using a logistic regression model with all potential confounders, ordinal categorizing the variables according to tertiles. The third tertile was the reference category except for kinetics, which is the change or decrease in each MDP value (related-sample difference between the first and second trimesters).

The statistical analysis was performed using SPSS statistical software package version 22.0. The level of statistical significance was set at 0.05 , and all tests were two-tailed.

This study was conducted according to the guidelines of the Declaration of Helsinki and was approved by the Clinical Research Ethics Committee of Cantabria (CEIC: 2020 - 428). Written informed consent was obtained from every subject.

\section{Results}

\subsection{Basal characteristics}

The baseline characteristics of pregnant women are summarized in Table 1. The mean age was $32.7 \pm$ 5.1 years, and the BMI was $25.2 \pm 5.0$ and $27.6 \pm 4.7 \mathrm{~kg} / \mathrm{m}^{2}$ in the first and second trimesters, respectively, with no significant differences between pregnant women with and without diabetes, except for a higher percentage of pregnant women who smoked in the GDM group: $25 \%$ vs $6.7 \%(p=0.038)$. 
Table 1

Characteristics of the study participants presented as the total study population and stratified according to gestational diabetes mellitus (GDM) status.

\begin{tabular}{|c|c|c|c|c|}
\hline \multirow[t]{2}{*}{ Variable } & Total & GDM & Non-GDM & \multirow[t]{2}{*}{$p$} \\
\hline & $(n=73)$ & $(n=28)$ & $(n=45)$ & \\
\hline Age, $(y r)($ mean $\pm S D)$ & $32.7 \pm 5.1$ & $32.8 \pm 5.4$ & $32.6 \pm 4.9$ & 0.937 \\
\hline \multicolumn{5}{|c|}{$\mathrm{BMI}\left(\mathrm{kg} / \mathrm{m}^{2}\right)($ mean $\pm \mathrm{SD})$} \\
\hline First Trimester & $25.2 \pm 5.0$ & $25.4 \pm 5.6$ & $25.0 \pm 4.7$ & 0.851 \\
\hline Second Trimester & $27.6 \pm 4.7$ & $27.9 \pm 5.0$ & $27.4 \pm 4.6$ & 0.704 \\
\hline \multicolumn{5}{|l|}{ Race/ethnicity } \\
\hline Non-Hispanic white & 70 (95.8\%) & $28(100 \%)$ & $42(93,3 \%)$ & \multirow[t]{3}{*}{0.565} \\
\hline African & $1(1.3 \%)$ & & $1(2.2 \%)$ & \\
\hline Hispanic & $2(2.7 \%)$ & & $2(4.4 \%)$ & \\
\hline \multicolumn{5}{|l|}{ Previous pregnancies } \\
\hline None n (\%) & $28(38.4 \%)$ & $10(35.7 \%)$ & $18(40.0 \%)$ & \multirow[t]{3}{*}{0.935} \\
\hline $1 \mathrm{n}(\%)$ & $30(41.1 \%)$ & $12(42.9 \%)$ & $18(40.0 \%)$ & \\
\hline +1 n (\%) & $15(20.5 \%)$ & $6(21.4 \%)$ & $9(20.0 \%)$ & \\
\hline Tobacco (yes) & $10(13.7 \%)$ & $7(25.0 \%)$ & $3(6.7 \%)$ & 0.038 \\
\hline \multicolumn{5}{|c|}{ Gestational age (weeks) (mean \pm SD) } \\
\hline First Trimester & $10.37 \pm 0.77$ & $10.42 \pm 0.57$ & $10.33 \pm 0.87$ & 0.262 \\
\hline Second Trimester & $25.09 \pm 1.45$ & $25.17 \pm 1.38$ & $25.04 \pm 1.50$ & 0.623 \\
\hline
\end{tabular}

\subsection{Humanin and MOTSc MDP levels in the first and second trimesters}

We found a significant decrease in humanin and MOTSc levels from the first to the second trimester of gestation (Table 2). The behavior of humanin was the same in pregnant women who later developed GDM and those who did not (Fig. 1a), but that of MOTSc was different, with a significant decrease only in the GDM group ( $p=0.012)$ and a nonsignificant decrease in the nondiabetes group $(p=0.076)$ (Fig. 1b). In one pregnant woman, humanin levels were abnormally high in the second trimester. The result was repeated and remained abnormally high. The results obtained by excluding this subject in a sensitivity analysis showed less difference in the comparison of kinetics between groups with no other significant 
differences. Supplementary Table S1 compares the humanin or MOTSc levels between pregnant women with and without GDM for each trimester of pregnancy separately.

Table 2

Evolution of mitochondrial-derived peptides, HOMA-IR and HOMA- $\beta$ between the first and second trimesters of gestation.

\begin{tabular}{|llll|}
\hline & First Trimester & Second Trimester & $p$ \\
\hline Humanin $(\mathrm{pg} / \mathrm{mL})$, mean $(\mathrm{SD})$ & $797.9 \pm 607.7$ & $697.2 \pm 523.0$ & $<0.001$ \\
\hline MOTSc $(\mathrm{ng} / \mathrm{mL})$, mean $(\mathrm{SD})$ & $725.1 \pm 332.8$ & $592.0 \pm 250.5$ & 0.003 \\
\hline Glucose $(\mathrm{mmol} / \mathrm{l})$, mean $(\mathrm{SD})$ & $4.3 \pm 0.3$ & $4.4 \pm 0.6$ & 0.400 \\
\hline Insulin $(\mu \mathrm{U} / \mathrm{mL})$, mean $(\mathrm{SD})$ & $8.2 \pm 4.5$ & $11.1 \pm 10.1$ & 0.001 \\
\hline HOMA-IR, mean $(\mathrm{SD})$ & $1.6 \pm 0.9$ & $2.3 \pm 2.5$ & 0.006 \\
\hline HOMA- $\beta(\%)$, mean $(\mathrm{SD})$ & $223.2 \pm 144.1$ & $288.1 \pm 262.0$ & 0.039 \\
\hline $\begin{array}{l}\text { MDPs: Mitochondrial-derived peptides. Related-sample comparisons across trimesters were } \\
\text { performed by the Wilcoxon test. }\end{array}$ & & \\
\hline
\end{tabular}

\subsection{Association with insulin resistance}

AUC values $\leq 0.705$ for both humanin and MOTSc peptides were obtained in the first and second trimesters separately for the risk of a high HOMA-IR index ( $\geq$ p70) (Fig. 2a) as well as for the samplerelated change for each peptide throughout pregnancy (Fig. 2b).

We found a statistically significant crude association between low levels of humanin (below median) and a higher risk of presenting a high HOMA-IR index ( $\geq$ p70) (Supplementary Table S2) in both the first and second trimesters of gestation, with a statistically significant linear $p$-trend when ordinal categorizing according to tertiles: OR for the lowest values of humanin in first trimester $=7.22$ (95\% Cl 1.70-30.64), linear $\mathrm{p}$-trend $=0.006$; OR for the lowest values of humanin in second trimester $=7.00(95 \% \mathrm{Cl} 1.67-$ 29.35), linear $p$-trend $=0.006$ (Table 3 ). We identified a negative correlation between BMI and humanin in both the first ( $r$ value $-0.343 ; p=0.003$ ) and second trimesters ( $r$ value $-0.358 ; p=0.006)$ (data not shown in tables). In this sense, after including BMI in the multivariate regression model in addition to maternal age and smoking habit, the association with HOMA-IR decreased in strength and lost statistical significance: adjusted OR for the lowest values of humanin $=2.63$ and 3.14 , linear $p$-trend $=0.260$ and 0.175 for the first and second trimesters, respectively (Table 3 ). 
Table 3

Association between levels of mitochondrial-derived peptides and the HOMA-IR index in the first and second trimesters of gestation.

\begin{tabular}{|c|c|c|c|c|c|c|c|c|c|}
\hline & & $\begin{array}{l}\text { HOMA- } \\
\text { IR }\end{array}$ & $\begin{array}{l}\text { HOMA- } \\
\text { IR high }\end{array}$ & & & & & & \\
\hline & & low & & & & & & & \\
\hline \multirow[t]{2}{*}{ MDPs } & \multirow[t]{2}{*}{$\begin{array}{l}\text { Cutoff } \\
\text { points }\end{array}$} & $\begin{array}{l}N=48 \\
(1 T)\end{array}$ & $\begin{array}{l}N=24 \\
(1 \mathrm{~T})\end{array}$ & \multirow{2}{*}{$\begin{array}{l}\text { Crude } \\
\text { OR }\end{array}$} & \multirow[t]{2}{*}{ (95\% } & \multirow[t]{2}{*}{ Cl) } & \multirow[t]{2}{*}{$\mathrm{aOR}^{\mathrm{a}}$} & \multirow[t]{2}{*}{$(95 \%$} & \multirow[t]{2}{*}{$\mathrm{Cl})$} \\
\hline & & $\begin{array}{l}N=49 \\
(2 \mathrm{~T})\end{array}$ & $\begin{array}{l}N=23 \\
(2 T)\end{array}$ & & & & & & \\
\hline \multicolumn{10}{|l|}{$\begin{array}{l}\text { First trimester } \\
\text { Humanin } \\
\text { (pg/mL) } \\
\text { (Tertiles) }\end{array}$} \\
\hline High (reference) & $790+$ & 20 & 3 & 1.00 & - & & 1.00 & - & \\
\hline Medium & $\begin{array}{l}567- \\
789\end{array}$ & 16 & 8 & 3.33 & 0.76 & 14.65 & 2.22 & 0.45 & 10.98 \\
\hline Low & $<=566$ & 12 & 13 & 7.22 & 1.70 & 30.64 & 2.63 & 0.51 & 13.43 \\
\hline $\mathrm{p}$ linear trend & & & & 0.006 & & & 0.260 & & \\
\hline \multicolumn{10}{|l|}{$\begin{array}{l}\text { First trimester } \\
\text { MOTSc }(\mathrm{ng} / \mathrm{ml}) \\
\text { (Tertiles) }\end{array}$} \\
\hline High (reference) & $823.3+$ & 16 & 7 & 1.00 & - & & 1.00 & - & \\
\hline Medium & $\begin{array}{l}526.9- \\
823.2\end{array}$ & 15 & 10 & 1.52 & 0.46 & 5.04 & 1.23 & 0.29 & 5.10 \\
\hline Low & $\begin{array}{l}<= \\
526.8\end{array}$ & 17 & 7 & 0.94 & 0.27 & 3.29 & 1.02 & 0.24 & 4.23 \\
\hline $\mathrm{p}$ linear trend & & & & 0.918 & & & 0.992 & & \\
\hline \multicolumn{10}{|l|}{$\begin{array}{l}\text { Second } \\
\text { trimester } \\
\text { Humanin } \\
\text { (pg/mL) } \\
\text { (Tertiles) }\end{array}$} \\
\hline High (reference) & $648+$ & 21 & 3 & 1.00 & - & & 1.00 & - & \\
\hline
\end{tabular}

Note: One case was excluded because of missing insulin values in the first trimester, and one control was excluded because of missing glucose values in the second trimester.

High HOMA-IR was considered $\geq 70$ th percentile of its distribution.

a aOR and $95 \% \mathrm{Cl}$ adjusted for maternal age, $\mathrm{BMI}$ and smoking habit. 


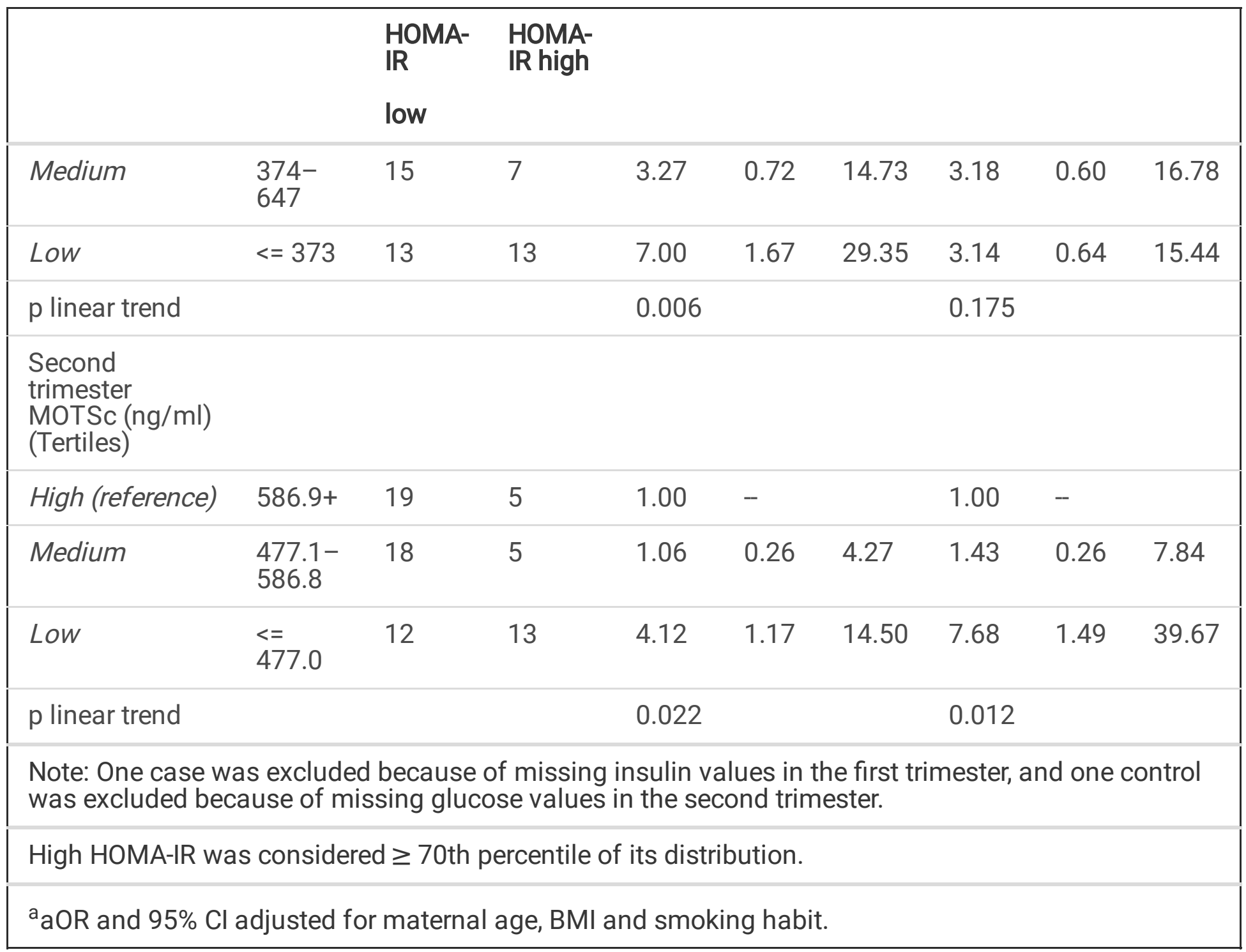

Regarding MOTSc, no association was identified between its levels and a high HOMA-IR index in the first trimester (Supplementary Table S2 and Table S3). However, in the second trimester, an association between low MOTSc levels and a higher risk of elevated HOMA-IR index was observed: adjusted OR for the lowest values of MOTSc $=7.68(95 \% \mathrm{Cl} 1.49-39.67)$, linear p-trend $=0.012$ (Table 3).

Regarding the evolution of MDPs throughout pregnancy, a positive correlation between a greater decrease in MOTSc levels throughout pregnancy and higher HOMA-IR in the second trimester of pregnancy was observed ( $r$ value $0.262 ; p=0.026$ ) (Supplementary Table S3). After adjusting the results for BMI, age and smoking status, pregnant women with a higher decrease in MOTSc levels presented a higher risk of elevated HOMA-IR index: adjusted OR 3.73 95\% Cl 1.03-13.50 ( $p=0.045)$ (Supplementary Table S4). No significant crude or adjusted associations were observed in relation to the change in humanin levels (Supplementary Table S4).

\subsection{Association with GDM}

Changes in MOTSc levels showed higher AUC levels among those analyzed in relation to the risk of developing GDM: AUC 0.576 (95\% Cl 0.442-0.710) (Supplementary Fig. S1). 
In the regression analysis, in consonance with the ROC approach, all the assessed parameters showed positive associations with the risk of gestational diabetes (adjusted OR $>1$ ), but they were of a small magnitude and did not reach statistical significance (Supplementary Table S5).

\section{Discussion}

We observed a significant decrease between the first and second trimesters of gestation in both humanin and MOTSc levels. The decrease in humanin was comparable among pregnant women who later developed GDM and those who did not. However, the decrease in MOTSc was only significant in the group of pregnant women who developed diabetes.

To our knowledge, there are no published studies on the plasma levels of MDPs (Humanin and MOTSc) in the early stage of gestation and their evolution throughout pregnancy. Furthermore, only one previous study has evaluated humanin levels during pregnancy with a single determination between 24-28 weeks [10] and no study has focused on MOTSc levels during pregnancy.

We also explored the relationship between MDPs and insulin resistance during early gestation. We found an association between low levels of humanin and a higher risk of presenting a high HOMA-IR index ( $\geq$ p70) in both the first and second trimesters of gestation; however, this association decreased in strength and lost statistical significance after adjusting the analysis by BMI. In fact, the identification of a negative correlation between the levels of humanin and BMI in both trimesters reinforces the hypothesis that BMI is an important confounder when interpreting the relationship between humanin and insulin sensitivity. Regarding MOTSc, surprisingly, the findings were not the same in the first trimester, where we did not identify a significant association with the HOMA-IR index and the second trimester, where we observed a significant relationship between the MOTSc levels and the HOMA-IR index with a higher risk of presenting a HOMA-IR index above p70 in pregnant women with lower MOTSc levels. This association was maintained after adjusting the analysis for both BMI and age, and it was mainly derived from a higher risk among pregnant women with MOTSc levels in the lower tertile. In addition, a more pronounced decline in MOTSc levels between the first and second trimesters resulted in a higher risk of having a HOMA-IR index above p70. Therefore, our results suggest that MOTSc levels, especially the decrease between the first and second trimesters of gestation, are associated with an increased risk of insulin resistance during early gestation. The correlation with the value of HOMA-IR in the second trimester, statistically significant although small, as well as the associations found when dichotomizing insulin resistance based on a cutoff point $\geq$ P70 of HOMA-IR, would support this hypothesis.

Our findings are consistent with previous studies that have identified a relationship between MDPs and insulin resistance. There is evidence of the insulin-sensitizing properties of MDPs mainly derived from cellular and animal models [11]. MOTSc is detected in the circulation, and its target organs are primarily skeletal muscle and fat. Administration of MOTSc in mice resulted in increased glucose uptake, primarily by skeletal muscle tissue, prevented the development of insulin resistance induced by a high-fat diet and reversed age-associated insulin resistance via activation of AMPK and SIRT1 [12]. Furthermore, MOTSc 
improves insulin sensitivity and increases beta-oxidation by targeting three metabolic pathways: sphingolipid metabolism, monoacylglycerol metabolism and dicarboxylate metabolism [13]. Humanin has been shown to decrease beta cell apoptosis in vitro and delay the development of diabetes in mouse NOD in vivo [14]. Finally, it has been described how people with type 2 diabetes mellitus have lower levels of humanin and MOTSc than people without diabetes, and their levels correlate with the HbA1c value [15].

Despite the relationship of MDPs with insulin sensitivity during pregnancy, their predictive capacity for the development of GDM was poor according to the AUC values obtained. This probably reflects the complexity of GDM, where insulin resistance is only one of the factors involved in its physiopathology. In fact, the ability to diagnose GDM in the first trimester remains controversial, and all parameters investigated have been poorly predictive of oral glucose tolerance test outcomes in the third trimester [16]. The highest precision achieved by a model defined as the summation of seven binary variables recommended by the National Institutes of Health $(\mathrm{NIH})$ was only $30 \%$, and its AUC for GDM was 0.682 [17].

Our study has several limitations. First, the small sample size could attenuate our ability to identify significant differences between pregnant women with and without GDM. Second, the single-center character of the study, and third the use of HOMA-IR as a marker of sensitivity to insulin. However, we consider that this mathematical model may be suitable to estimate the longitudinal changes in insulin sensitivity in our study population and has been shown to be an independent risk factor for the development of GDM [18]. Furthermore, a good correlation between HOMA-estimated insulin resistance and the euglycemic clamp [19] or minimal model [20] has been described. Further studies with larger sample sizes should extend and corroborate our results. In relation to the strengths of the study, it is worth mentioning the effort to study the associations through different analysis strategies, exploring also the dose-response pattern, and the control of confounding in the design phase through matching and by using multivariate analysis.

In conclusion, we found a significant decline in humanin and MOTSc levels between the first and second trimesters of pregnancy. The decrease in humanin was significant in pregnant women who developed diabetes and those who did not, while that of MOTSc was only significant in pregnant women who developed diabetes. Moreover, a greater decrease in MOTSc levels is associated with a higher risk of presenting a high HOMA-IR in the second trimester, while the relationship between humanin and HOMA-IR is attenuated and becomes nonsignificant after including BMI in the analysis. Thus, our results suggest that MOTSc levels, especially a strong decrease between the first and second trimesters of gestation, may be involved in the progressive increase in insulin resistance starting from early gestation.

\section{Declarations}

Funding 
This study did not receive any specific grant from any funding agency in the public, commercial, or notfor-profit sector

Conflict of interests

The authors have no potential conflicts of interest relevant to this article

Ethics approval

This study was conducted according to the guidelines of the Declaration of Helsinki and was approved by the clinical research ethics committee of Cantabria (CEIC: 2020-428).

Consent to participate

Written informed consent was obtained from every subject.

Availability of data and materials

The datasets generated during and/or analyzed during the current study are not publicly available but are available from the corresponding author upon reasonable request

Code availability

Not applicable

Author contributions

DR: conceptualization, data curation, formal analysis, investigation, methodology, project administration, resources, supervision, validation, visualization, writing-original draft, writing-review and editing; MS:

formal analysis, methodology, software, supervision, validation, visualization, writing-original draft, writing-review and editing; BA-L: data curation, project administration, resources, supervision, validation, visualization, writing-original draft, writing-review and editing; IA: data curation, investigation, resources, validation, writing-review and editing; $A B$ : resources, validation, writing-review and editing; $C M$ : investigation, project administration, resources, software, supervision, validation, visualization, writingreview and editing; LAV: conceptualization, formal analysis, investigation, project administration, resources, supervision, validation, visualization, writing-review and editing.

\section{References}

1. Powe, C. E., Presley, L. P. H., Locascio, J. J. \& Catalano, P. M. Augmented insulin secretory response in early pregnancy. Diabetologia. 62, 1445-1452 (2019). https://doi.org/10.1007/s00125-019-4881-6

2. World Health Organization. Definition, diagnosis and classification of diabetes mellitus and its complications. Part 1: diagnosis and classification of diabetes mellitus. World Health Organization, Geneva. (1999). 
3. Metzger, B.E. et al. International association of diabetes and pregnancy study groups recommendations on the diagnosis and classification of hyperglycemia in pregnancy. Diabetes Care. 33, 676-682 (2010). https://doi.org/10.2337/dc09-1848

4. American Diabetes Association. Classification and diagnosis of diabetes: standards of medical care in diabetes-2020. Diabetes Care. 43, S14-S31 (2020). https://doi.org/10.2337/dc20-S002

5. Sweeting, A. N. et al. Gestational diabetes mellitus in early pregnancy: evidence for poor pregnancy outcomes despite treatment. Diabetes Care. 39, 75-81 (2016). https://doi.org/10.2337/dc15-0433

6. Diaz-Vegas, A. et al. Is mitochondrial dysfunction a common root of noncommunicable chronic diseases? Endocr Rev. 41, 491-517 (2020). https://doi.org/10.1210/endrev/bnaa005

7. McElwain, C. \& McCarthy, C. M. Investigating mitochondrial dysfunction in gestational diabetes mellitus and elucidating if BMI is a causative mediator. Eur J Obstet Gynecol Reprod Biol. 251, 6065 (2020). https://doi.org/10.1016/j.ejogrb.2020.04.037

8. Popov, L.D. Mitochondrial peptides-appropriate options for therapeutic exploitation. Cell Tissue Res. 377,161-165 (2029). https://doi.org/10.1007/s00441-019-03049-z

9. Nashine, S. \& Kenney M. C. Effects of mitochondrial-derived peptides (MDPs) on mitochondrial and cellular health in AMD. Cells. 9,1102 (2020). https://doi.org/10.3390/cells9051102

10. $\mathrm{Ma}, \mathrm{Y}$. et al. Comparison of serum concentrations of humanin in women with and without gestational diabetes mellitus. Gynecol Endocrinol. 34, 1064-1067 (2018).

https://doi.org/10.1080/09513590.2018.1482869

11. Merry, T. L., Chan, A., Woodhead, J. S. T., Reynolds, J. C., Kumagai, H., Kim, S. J. \& Lee, C. Mitochondrial-derived peptides in energy metabolism. Am J Physiol Endocrinol Metab. 319, E659E666 (2020). https://doi.org/10.1152/ajpendo.00249.2020

12. Lee, C. et al. The mitochondrial-derived peptide MOTS-c promotes metabolic homeostasis and reduces obesity and insulin resistance. Cell Metab. 21, 443-454 (2015). https://doi.org/10.1016/j.cmet.2015.02.009

13. Kim, S. J. Et al. The mitochondrial-derived peptide MOTS-c is a regulator of plasma metabolites and enhances insulin sensitivity. Physiol Rep. 7:e14171 (2019). https://doi.org/10.14814/phy2.14171

14. Hoang, P. T. et al. The neurosurvival factor Humanin inhibits beta-cell apoptosis via signal transducer and activator of transcription 3 activation and delays and ameliorates diabetes in nonobese diabetic mice. Metabolism. 59, 343-349 (2010). https://doi.org/10.1016/j.metabol.2009.08.001

15. Ramanjaneya, M. et al. Mitochondrial-derived peptides are down regulated in diabetes subjects. Front Endocrinol (Lausanne). 10:331 (2019). https://doi.org/10.3389/fendo.2019.00331

16. Mclntyre, H. D. et al. Issues with the diagnosis and classification of hyperglycemia in early pregnancy. Diabetes Care. 39, 53-54 (2016). https://doi.org/10.2337/dc15-1887

17. Artzi, N. S. et al. Prediction of gestational diabetes based on nationwide electronic health records. Nat Med. 26, 71-76 (2020). https://doi.org/10.1038/s41591-019-0724-8 
18. Badon, S. E. et al. A pre-pregnancy biomarker risk score improves prediction of future gestational diabetes. J Endocr Soc. 2, 1158-1169 (2028). https://doi.org/10.1210/js.2018-00200

19. Matthews, D. R., Hosker, J. P., Rudenski, A. S., Naylor, B. A., Treacher, D. F. \& Turner, R. C. Homeostasis model assessment: insulin resistance and beta-cell function from fasting plasma glucose and insulin concentrations in man. Diabetologia. 28, 412-419 (1985).

https://doi.org/10.1007/bf00280883

20. García-Estévez, D. A., Araújo-Vilar, D., Fiestras-Janeiro, G., Saavedra-González, A. \& Cabezas-Cerrato J. Comparison of several insulin sensitivity indices derived from basal plasma insulin and glucose levels with minimal model indices. Horm Metab Res. 35, 13-17 (2003). https://doi.org/10.1055/s2003-38385

\section{Figures}
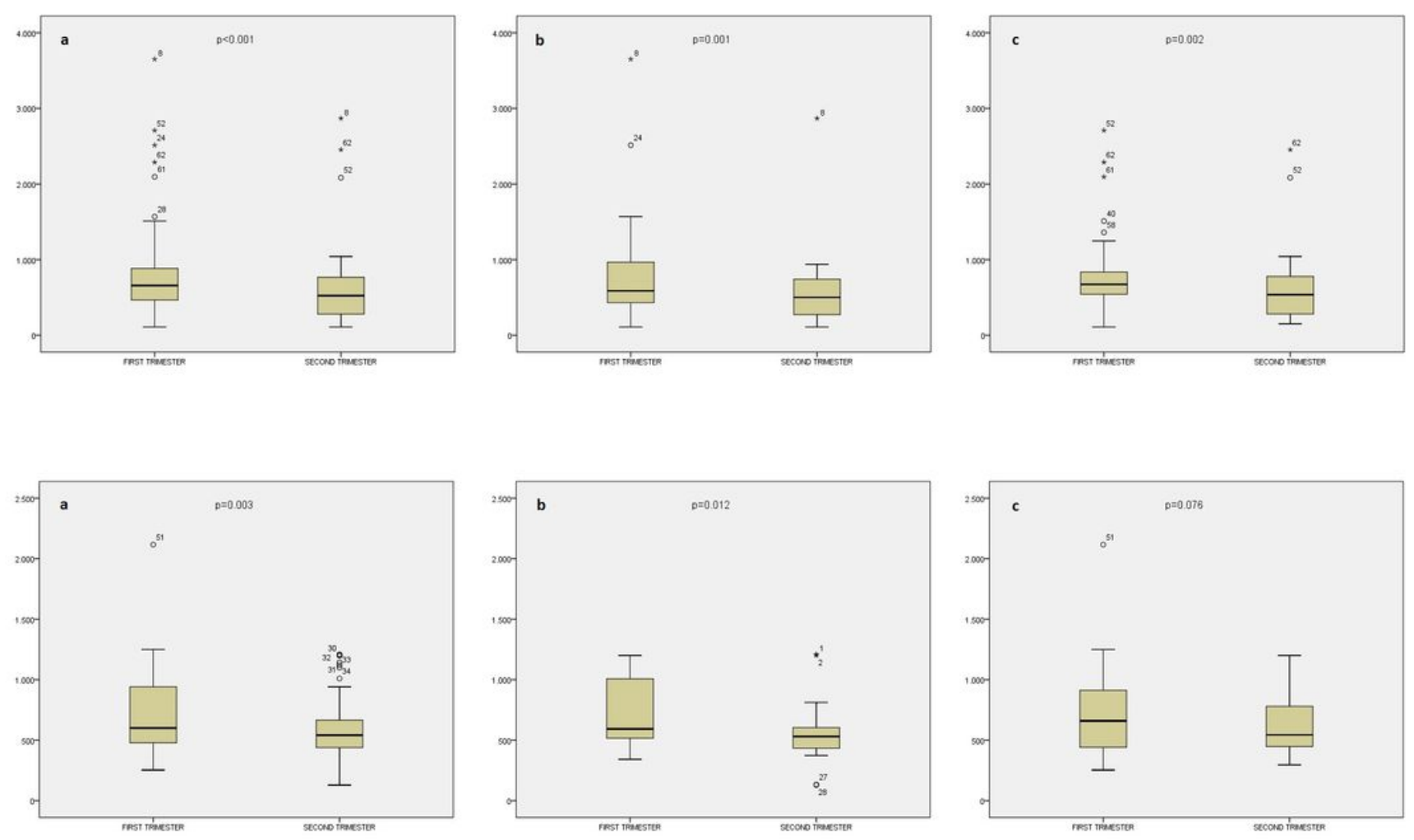

\section{Figure 1}

a. Box plots of Humanin levels in the first and second trimesters in the total cohort (a) and restricted to women with gestational diabetes (b) and controls (c). The box-plots represent lines and boxes represent 
the median and interquartile range and whiskers calculate outlier data. The Wilcoxon test was used for the Related-Samples comparisons between trimesters. Fig. 1b. Box plots of MOTSc levels in the first and second trimesters in the total cohort (a) and restricted to women with gestational diabetes (b) and controls (c). The box plots represent lines, boxes represent the median and interquartile range, and whiskers calculate outlier data. The Wilcoxon test was used for the related-samples comparisons between trimesters.
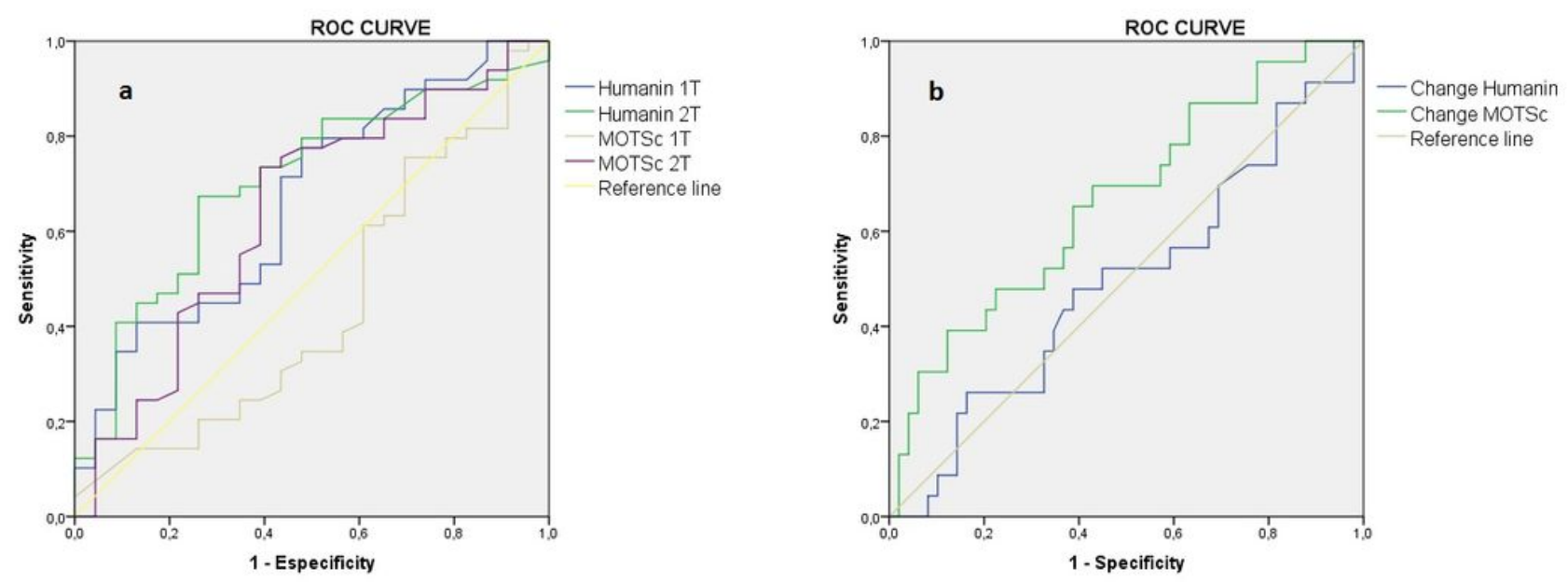

\section{Figure 2}

Receiver operating characteristic (ROC) curve for high HOMA-IR in relation to mitochondrial-derived peptides in the first trimester (1T) and second trimester (2T) separately (a) and in relation to samplerelated changes for each peptide throughout pregnancy (b). Footnotes a High HOMA-IR was defined as a value $\geq$ percentile 70 Humanin 1T: AUC 0,698 (95\% Cl 0.569-0,826); Humanin 2T: AUC $0.705(95 \% \mathrm{Cl}$ 0.578-0.831); MOTSc 1T: AUC 0.497 (95\% Cl 0.355-0.638); MOTSc 2T AUC: 0.640 (95\% Cl 0.498-0.782). b High HOMA-IR was defined as a value $\geq$ percentile 70 Change in Humanin: AUC 0.494 (95\% Cl 0.3480.640) Change in MOTSc: AUC 0.665 (95\% Cl 0.531-0.800)

\section{Supplementary Files}

This is a list of supplementary files associated with this preprint. Click to download.

- Supplementarymaterial.pdf 